\title{
UNA MIRADA HACIA EL FUTURO DE LA EDUCACIÓN EN LA UNIVERSIDAD DE CHILE
}

Víctor Pérez Vera 


\section{VICTOR PÉREZ VERA}

Ingeniero civil industrial de la Universidad de Chile (1968). En 1972 obtuvo un Master of Science en Ingeniería Industrial en la Universidad de Michigan (Estados Unidos). En 1969 fue nombrado profesor investigador del Departamento de Ingeniería Industrial en la Facultad de Ciencias Físicas y Matemáticas, facultad en la que también ocupó los cargos de director del Departamento de Ingeniería Industrial (1980-1984 y 1988-1990), Vicedecano (1984-1985), miembro de la Comisión de Evaluación Académica (1991-1992) y decano (1994-2002). En 1993 ocupó la Prorrectoría de la Universidad de Chile. Ha publicado numerosos trabajos en revistas internacionales de corriente principal, en el área de los sistemas de información. Es autor y coautor de siete libros sobre informática, sistemas de información, bases de datos y análisis financiero, todos editados por la Editorial Universitaria. En 2010 fue electo rector de la Universidad de Chile por un segundo período. 


\section{UNA MIRADA HACIA EL FUTURO DE LA EDUCACIÓN EN LA UNIVERSIDAD DE CHILE}

\section{INTRODUCCIÓN}

Al cumplir nuestra Universidad 170 años y observar el decurso de su historia, es evidente que la impronta de la educación fue su norte y con la creación del Instituto Pedagógico, a fines del siglo XIX, un pivote que estructuró su quehacer. Los acontecimientos históricos del Golpe militar y el emplazamiento de la dictadura tuvieron consecuencias poco estudiadas en la Universidad de Chile, pero quienes fuimos testigos sabemos de las profundas consecuencias que ello implicó. Una, poderosa y cuyos efectos ha sufrido todo el país, fue el cercenamiento del Instituto Pedagógico, y con ello, la pérdida de una tradición en la educación de profesores, bajo los más altos estándares intelectuales, el pluralismo y el amor al conocimiento.

$\mathrm{Al}$ asumir, en junio del 2010, el segundo período como rector, y tal y como indicaba el programa que presenté a la comunidad universitaria, planteé que nuestro horizonte principal estaría enfocado a la recuperación del liderazgo en educación. La restitución de esa función tan cara a nuestra Universidad emergió como deber hacia Chile y su necesidad de contar con profesores y profesoras de primer nivel, bajo el sello de nuestra institución. Desde ese anuncio, la creación el Proyecto Institucional de Educación de la Universidad $^{1}$ se ha ido plasmando y dando cuerpo y realidad a las orientaciones estratégicas emanadas del Plan de Desarrollo Institucional aprobado por el Senado Universitario. Se trata de responder a los anhelos de cambio que reclama el sistema educativo chileno, elevar la valoración de la profesión docente y contribuir a la construcción de una cultura que reconozca que cada niño y niña chilenos son sujetos iguales en dignidad, derechos y oportunidades.

Reflexionar y colocar como perspectiva el tema de la educación en la Universidad de Chile y los anhelos y medios propuestos para recuperar el liderazgo en un terreno tan importante para los ciudadanos y para el crecimiento como país, resulta relevante de cara a los 170 años que cumple nuestra Universidad. Potenciar la idea de crear una FACULTAD DE EDUCACIÓN, avanzando en su concreción, sus cimientos y sus sentidos epistemológicos, culturales y políticos, es un compromiso con nuestra misión y con el país.

Por eso, en este artículo nos parece oportuno difundir el contenido del trabajo que hemos realizado, y dar a conocer, más allá de los ámbitos estrictamente académicos,

1. Entre octubre del 2010 y enero del 2012, una comisión de académicos presididos por la prorrectora profesora Rosa Devés se encargó de conducir un proceso de reflexión y debate destinado a identificar las bases esenciales sobre las cuales la Universidad de Chile debiera sustentar y desarrollar el área académica y profesional de la educación. El resultado de este proceso se encuentra en el Informe "Bases para el Proyecto Institucional de Educación de la Universidad de Chile". 
los procesos de investigación seguidos y los resultados obtenidos en el camino de la construcción del proyecto de restablecimiento de la Educación en la Universidad de Chile.

\section{HACIA UN PROYECTO INSTITUCIONAL DE EDUCACIÓN DE LA UNIVERSIDAD DE CHILE}

El 23 de junio de 2010 señalamos en nuestro discurso de asunción al cargo que:

Sustentaremos los esfuerzos del país por mejorar la calidad y equidad de la educación. Pondremos todos nuestros recursos humanos e intelectuales para impulsar un gran proyecto institucional educativo que considere la investigación y creación en educación, la formación inicial de profesores en los niveles preescolar, básico y medio, así como los programas de posgrado y de educación continua, todo según los más exigentes estándares internacionales. Nuestra meta debe ser recuperar para la Universidad de Chile el liderazgo nacional en educación. Tenemos que llegar al aula de todos los colegios del país, sobre todo a los de la educación pública de los sectores más vulnerables, con nuestros conocimientos y valores. Es en el aula de esos colegios donde se define el tipo de país futuro que tendremos. El objetivo esencial de esta iniciativa es la preservación de la educación pública de calidad. Para abordar este desafío, recurriremos a los académicos y académicas de las distintas unidades que en la actualidad se encuentran involucrados activamente en programas de formación inicial de profesores, investigación en educación y educación continua.

La consideración de otros aspectos, como que la cantidad de proyectos FONDECYT referidos a educación han ido en aumento en estos últimos años, o la importancia que se ha dado a la calidad de la docencia universitaria y a la producción de evidencia para su mejora, indican que el interés por esta área ha ido en aumento y ha dado sus frutos. La reconstrucción de esta breve historia de los últimos 30 años, sirve para observar que siempre se ha tratado de una materia de suma importancia y consideración dentro de las carreras docentes.

Sin embargo, conjeturamos que podrían existir también algunos aspectos subjetivos que han conducido al insatisfactorio resultado actual: inicialmente pudo producirse un sentimiento de castigo con el cercenamiento del Instituto Pedagógico; después, quizás la inercia o callejón sin salida que generó este acto, o la espera a que el daño fuera reparado; más tarde por falta de iniciativas estratégicas para crear una alternativa y, posteriormente, por el posible temor a la falta de calidad. Pero de la historia de estos últimos 30 años se puede extraer algunos de los elementos o temas que aparecen más recurrentes, y sobre todo bajo la óptica y el desafío de elaborar una Propuesta Institucional de Educación en la Universidad. 
1. Las iniciativas en la Universidad de Chile referidas a educación, y en especial a la formación de profesores, han sido históricamente el resultado de una "interpelación" principalmente desde el Estado hacia la Universidad, incluyendo en ello lo referido a decisiones institucionales de gran envergadura y a recursos, ya sea a través de tensiones democráticas o de resoluciones arbitrarias.

2. Las voluntades de política universitaria, las capacidades académicas, la calidad y contenidos de los programas e iniciativas han sido tópicos recurrentes en la discusión interna cada vez que el asunto ha sido abordado por parte de la Universidad en su conjunto. A nivel de iniciativas locales, ha sido claro que cuando ha existido voluntad de acometer cambios y de generar capacidades académicas, se han apreciado mejoras y se ha elevado la calidad; por el contrario, el abandono ha tendido a generar crisis, falta de proyectos y baja calidad.

3. Es bastante notorio que entre 1993 y 2010 ha existido en la Universidad de Chile una importante y creciente experiencia académica acumulada en educación y en formación de profesores; sobre todo a partir de un involucramiento ascendente, aunque dispar y no siempre continuo. No obstante, a pesar de la aparición de definiciones estratégicas relevantes en este mismo período, dicho involucramiento se ha manifestado fundamentalmente de manera dispersa a través de diferentes unidades y acciones. Es decir, no ha logrado configurar un proyecto de desarrollo, ya sea que este emerja como resultado de las diferentes iniciativas, o bien que estas sean el resultado de aquel. Esto se vincula con una dificultad estructural en la Universidad de Chile para que la coordinación y convergencia entre unidades académicas en pos de proyectos institucionales de gran alcance se desarrolle de forma espontánea.

Las diferentes iniciativas y momentos aquí ilustrados, así como las voluntades declaradas en sucesivos momentos, tienden a configurar un camino donde la tendencia es a establecer una definición de orden mayor. Así, en la actualidad la Universidad de Chile se encuentra, por su propia historia pasada y reciente, por su Misión, y por la propia sociedad chilena y las necesidades que esta manifiesta, "interpelada" a realizar definiciones explícitas y de orden estratégico en el área de la educación y de formación de profesores. Sin embargo, el análisis de los hitos ocurridos desde 1990 y el recorrido que estos configuran, sugieren que su desarrollo en la Universidad de Chile, pensado a una escala "mayor" -mayor en calidad, cantidad, articulación, visibilidad, impacto y sustentabilidad-es inviable desde la sola emergencia de iniciativas dispersas, dispares y sin decisiones institucionales cualitativamente superiores que comprometan al conjunto de la Universidad. Es decir, requiere también una decisión de Estado.

El proceso de análisis y definición sobre las Bases para el Proyecto Institucional de Educación de la Universidad de Chile, desarrollado en 2011 y presentado a inicios del año pasado, representa en este sentido una oportunidad histórica para delinear el salto cualitativo que la Universidad ha estado reclamándose en las últimas décadas. 


\section{PROYECTO INSTITUCIONAL DE LA EDUCACIÓN EN LA UNIVERSIDAD DE CHILE}

Como se puede apreciar, desde mediados de los 90, pero con mayor intensidad desde el 2000, las iniciativas en educación de la Universidad de Chile han surgido en diferentes espacios institucionales acumulando experiencias y capacidades significativas que han tendido a cubrir el espectro de funciones básicas asociadas con la labor universitaria. Nosotros, desde el 2006, nos hemos orientado a la continuidad del trabajo en esta línea, potenciando en ambos casos planes y estrategias que conduzcan hacia la creación de una Facultad de Ciencias de la Educación.

A los hitos significativos realizados en estos últimos seis años, y que ya expusimos, habría que añadir el conjunto de actividades y trabajos desarrollados (a nivel nacional como a nivel internacional y tanto sobre la formación inicial como sobre la formación continua del profesorado) por los distintos departamentos, comisiones y equipos, que fueron creados durante esta etapa para investigar sobre las tendencias y estrategias del panorama mundial y análisis nacional en el área de educación.

\section{El compromiso institucional con el desarrollo de la educación}

En el Prefacio a las Bases para el Proyecto Institucional de Educación de la Universidad de Chile (2011), sostuvimos:

Diferentes razones llaman hoy a la Universidad de Chile a involucrarse a una escala mayor en el campo de la educación y en particular en la formación de profesores: desde el enorme desafío intelectual, académico y profesional que aquello representa, hasta la voz legitima que nuestra Universidad y su comunidad encarnan hoy por la defensa y desarrollo de una educación pública de calidad en el país; pasando por nuestra propia historia institucional hasta llegar a nuestros deberes y responsabilidades para con el presente y futuro de los chilenos y chilenas.

En este contexto, nuestra rectoría decidió convocar a un proceso de definición diseño e implementación para el Proyecto Institucional de Educación de la Universidad, con el fin de dotar de cuerpo y realidad a las orientaciones estratégicas emanadas del Plan de Desarrollo Institucional aprobado por el Senado y el Consejo Universitarios.

Esencialmente se trata de responder, desde nuestro oficio, al cambio estructural que Chile reclama para su sistema educativo, y aportar con ello, a cimentar cambios culturales de largo aliento, entre otros, a elevar la valoración de la profesión docente y contribuir a la construcción de una cultura que reconozca en cada niño y niña chileno un sujeto igual en dignidad, derechos y oportunidades.

Así, la convocatoria que hemos hecho a la Universidad de Chile en su conjunto tiene por objetivo dar pasos decididos con el fin de realzar la excelencia de la formación docente en todos los niveles de enseñanza: parvularia, básica y media. 
El compromiso de potenciar el área de educación en la Universidad de Chile ha sido expresado públicamente por nosotros en diversas oportunidades. En octubre de 2010, cuando convocamos a la Comisión de Proyecto Institucional de Educación con la tarea de conducir la primera fase de análisis y pre diseño, tuvimos en cuenta los siguientes objetivos específicos:

1. Elaborar un modelo educativo para las pedagogías y realizar una propuesta para asegurar programas de formación inicial y continua de profesores de excelencia en todos los niveles (preescolar, básica y media), a través del fortalecimiento de los programas existentes y de la creación de programas nuevos. Esto requiere actualizar los programas de estudio, modernizar la infraestructura y el equipamiento, incorporar nuevo personal académico de excelencia y fortalecer la gestión institucional.

2. Promover y proponer acciones para robustecer y elevar la investigación en educación a un estándar de calidad internacional, especialmente aquella investigación relevante para la formación docente y evaluar las capacidades existentes y proponer medidas para el desarrollo del posgrado en educación, teniendo como meta la formación a nivel doctoral. Se deberá generar sinergias para vincular el pregrado con el posgrado y la investigación, aportando a una mejor comprensión de los procesos enseñanza-aprendizaje en el contexto del sistema educativo.

3. Proponer una institucionalidad universitaria coherente con el Proyecto Institucional de Educación, capaz de resguardar los mencionados principios de excelencia académica, integración disciplinaria y organizacional, vocación pública, visión de futuro y apertura al mundo.

4. Estimar las inversiones necesarias para dotar de la infraestructura y del cuerpo académico que se requiere para el logro de las metas establecidas en el Proyecto Institucional de Educación.

En ese marco, la estrategia seguida por la Comisión de Proyecto Institucional de Educación apuntó, en primer lugar, a reunir e involucrar a la masa crítica académica de la Universidad de Chile que trabaja en torno a la educación, incluyendo tanto a académicos como estudiantes, buscando a la vez conocer y dialogar con la experiencia internacional y nacional en el área de formación docente. Participaron en diferentes esfuerzos y momentos de esta fase, académicos del Departamento de Estudios Pedagógicos (DEP) perteneciente a la Facultad de Filosofía y Humanidades, del Centro de Investigación Avanzada en Educación (CIAE), el Departamento de Educación de la Facultad de Ciencias Sociales (DE-FACSO) y de otras Unidades de la Universidad de Chile, como las Facultades de Ciencias, Ciencias Físicas y Matemáticas y Medicina.

En este contexto se realizaron encuentros con rectores y académicos nacionales y extranjeros, se llevaron a cabo encuentros específicos con estudiantes de los distintos programas de pedagogía de la Universidad: DEP, Licenciatura en Ciencias Exactas conducente al Título de Profesor en Matemáticas y Física y la actual carrera de Educación Parvularia y Básica Inicial. Se contó con la visita, conferencias e informes del profesor 
Graham Parr de la Universidad de Monash, Australia, y con conferenciantes diversos de otras universidades extranjeras. Se realizaron viajes de especialización y vinculación internacional a Australia, Cuba, Canadá, Estados Unidos, Finlandia, Inglaterra y Suecia, que permitieron conocer las experiencias de universidades extranjeras con vivencias relevantes en el área de educación y formación docente.

La Comisión también llevó a cabo talleres y conversaciones con profesores en ejercicio, provenientes de contextos diversos y diferentes niveles formativos. Parte de la estrategia de trabajo consideró, además, establecer vinculaciones y sinergias colaborativas con otros proyectos en curso en la institución, como son: el caso del Convenio de Desempeño Iniciativa Bicentenario Campus Juan Gómez Millas, los Proyectos Estándares de Formación de Profesores de Enseñanza Básica en Lenguaje y Matemáticas para la Formación Parvularia, la Red para la Excelencia docente, el proyecto de biología bidisciplinaria en Biología y Química, etc.

Finalmente, se realizaron diferentes trabajos de análisis y diagnóstico que permitieron avanzar en cinco aspectos relevantes:

1. Definir las bases conceptuales de los futuros programas de formación inicial docente en Educación parvularia, básica y media, teniendo presentes cuatro grandes ámbitos: a) los desafíos nacionales en el área de la educación, con especial foco en la formación docente; b) la experiencia de la Universidad en el desarrollo de programas de formación inicial de calidad y en la investigación asociada; c) las limitaciones de los programas de formación inicial existentes en nuestra institución y otras universidades del país; d) un análisis de experiencias internacionales relevantes.

2. Reconocer las capacidades en investigación educacional presentes en las diferentes unidades de la Universidad de Chile (personas, áreas temáticas, proyectos).

3. Evaluar las capacidades existentes a nivel del posgrado en educación y establecer horizontes para su desarrollo, incluida la articulación pregrado-posgrado y el desarrollo de la formación continua y de postítulo.

4. Identificar y reconocer las principales limitaciones a nivel institucional y las condiciones mínimas necesarias para un desarrollo del área educación de la formación docente en la Universidad de Chile.

5. Definir los principales lineamientos para la nueva institucionalización de la formación docente y de la educación como área específica en la Universidad de Chile.

Estos aspectos implican avanzar en:

- La creación de una nueva oferta académica de pregrado para la formación inicial docente que contemple nuevos programas en los niveles de educación parvularia, básica y media.

En el mediano plazo se propone avanzar en contar con siete programas diferentes: Educación básica a partir del primer año de ingreso a la Universidad (modalidad concurrente) 
y también a partir de una licenciatura (modalidad consecutiva); educación parvularia desde el primer año de ingreso; educación media en las áreas de Humanidades, Ciencias y Artes (en la modalidad consecutivo-integrada), es decir, con participación principal de dos unidades académicas, una con foco en la disciplina a enseñar en el sistema escolar y otra con foco en la disciplina pedagógica. Al final del artículo nos referiremos a estos tres modelos de formación.

Para hacer posible la consecución de estos objetivos, se considera necesaria la creación de una nueva unidad académica en la Universidad de Chile que tenga su foco principal en la educación como disciplina y que concentre labores de pregrado, posgrado, investigación, extensión y vinculación con el medio.

Para cumplir con sus objetivos, esta nueva unidad académica especializada en educación deberá dotarse de una estructura organizativa flexible y liviana, con un equipo directivo y académico de alto nivel, así como un modelo de gestión eficiente.

El proceso de instalación de esta unidad académica especializada en educación deberá ser gradual y deberá considerar para su éxito los siguientes aspectos:

- Marcar los hitos fundacionales que distinguirán a esta unidad de otras realidades preexistentes en la Universidad y en el país.

- Conformar los equipos académicos que liderarán el cambio y definir los planes de desarrollo e implementación que se correspondan con ello.

- Facilitar el tránsito desde la situación actual de la dispersión y disparidad, en que distintas unidades académicas desarrollan programas y proyectos en el ámbito de la educación, hacia un accionar integral, orgánico y articulado del área de educación y formación docente en la Universidad de Chile.

\section{Voluntad para asumir el desafío}

El conjunto de los hitos y procesos mencionados demuestran una voluntad creciente por desarrollar el área de educación en la Universidad de Chile. Esta evolución se ve forzada en la actualidad por el compromiso que hemos asumido para que la Universidad de Chile construya un Proyecto Institucional de Educación acorde con las necesidades del país.

De esta manera nuestra institución retoma el desafío y la responsabilidad de ser un actor relevante en uno de los campos más complejos y estratégicos para la sociedad del conocimiento, conforme a las orientaciones establecidas en su Misión y concibiendo los procesos educacionales en sentido amplio, como parte del desarrollo cultural, social y económico de las sociedades humanas.

$\mathrm{Al}$ adquirir este reto, la Universidad de Chile quiere responder a las necesidades de Chile en materia educacional. Por lo mismo, su contribución en el campo de la educación estará guiada por la responsabilidad con lo público y el compromiso con la calidad y la equidad educacionales. Su aporte se basará fundamentalmente en la generación y diseminación de nuevos conocimientos y en la formación docente inicial y continua de calidad. 
De este modo, la Universidad de Chile busca contribuir, especialmente, a:

- El desarrollo humano del país, a través de la elevación de las capacidades de su población y la expansión de sus bases intelectuales para el acceso y apropiación del conocimiento.

- La formación de ciudadanía, la ampliación de las libertades y la profundización de la democracia.

- La inclusión y cohesión social, y la distribución equitativa de las oportunidades.

- Las reformas estructurales y la mejora constante del sistema escolar chileno, tanto desde su propio desarrollo histórico como desde una perspectiva comparada.

- El análisis, comprensión, desarrollo e innovación de los procesos educativos en todos los niveles y formas.

- Resolver los problemas y desafíos concretos que enfrentan las comunidades educacionales, especialmente aquellas que enfrentan mayores desventajas.

Para el cumplimiento de estos propósitos, debemos generar conocimiento riguroso en el campo de la investigación en educación, contribuir a las políticas públicas y a los procesos de cambio en el sistema escolar -así como al mejoramiento educacional y a la innovación a través de proyectos de investigación y desarrollo- y conocer y dialogar de manera permanente con la experiencia internacional en educación en general y en formación docente en particular.

Este horizonte requiere de recursos humanos y materiales de alto nivel en el área de la educación y de la consolidación de una cultura académica integradora, creativa y de calidad, capaz de articular un diálogo fecundo, tanto dentro de la Universidad como entre esta y la sociedad. Ello implica un trabajo conjunto entre académicos con experiencias y visiones diversas y exige también una vinculación relevante y productiva entre áreas disciplinarias y unidades académicas nacionales e internacionales, y entre la Universidad y el sistema escolar.

\section{Hacia una nueva institucionalidad}

El desafío de instalar y recuperar a la educación como un área de primera importancia en la Universidad de Chile requiere de una nueva institucionalidad que le otorgue una base fundacional desde donde potenciar significativamente su desarrollo.

En efecto, el análisis de las decisiones institucionales referidas a educación y a la formación docente, así como de los procesos académicos en esta área durante los últimos 20 años, muestran de manera bastante clara (como hemos podido observar a lo largo de este texto) que sin la existencia de un espacio institucional que sirva de base al desarrollo del área, la dispersión institucional hace inviable dar un salto cualitativo en la materia. Más aun, sin dicho punto de apoyo, incluso mejoras incrementales respecto a lo hoy existente carecerían del impacto significativo que se busca, tanto dentro de la Universidad como, muy especialmente, hacia la sociedad. 
En consecuencia, habida cuenta de la experiencia internacional revisada y visitada, tanto del diagnóstico de las capacidades con que hoy cuenta la Universidad de Chile en educación y formación docente, así como de la identificación de las limitantes para su desarrollo, se proponen los siguientes ejes para la toma de decisiones sobre la nueva institucionalidad del área de educación:

- Crear una unidad académica en la Universidad de Chile que tenga su foco principal en la educación como disciplina y que concentre las labores de pregrado, posgrado, investigación, extensión y vinculación con el medio. Esta unidad funcionará en estrecha colaboración con otras unidades académicas de la Universidad, con establecimientos escolares del sistema escolar y con organizaciones externas relevantes en educación.

- Integrar a través de dicha unidad las capacidades existentes y desarrollar otras nuevas, asociando a un nivel superior la investigación en educación con la formación docente.

- Potenciar la investigación multidisciplinaria y vinculada tanto a los principales problemas de grandes grupos de la población, como a los desafíos de la educación nacional, atendiendo, además, a fenómenos emergentes y relevantes como el multiculturalismo, la equidad de género, la inclusión y la cohesión social, las TICs, etc.

- Asegurar que los futuros educadores, en especial quienes serán profesores de enseñanza media, reciban una formación disciplinaria, integrada y de calidad, en las distintas áreas formativas definidas por los perfiles de egreso, comprometiendo en ello a diferentes unidades académicas de la Universidad. Lo anterior es considerado como una condición necesaria para la excelencia académica de los programas de formación docente. Esta condición se construye a través de la participación y colaboración de las unidades académicas especializadas que cultivan las disciplinas involucradas, las cuales se articulan mediante el trabajo conjunto coordinado de cuerpos académicos multidisciplinarios que colaboran con el logro de los perfiles profesionales comprometidos. (Esta colaboración entre unidades no debe ser entendida, bajo ningún punto de vista, como una simple "prestación de servicios", so pena de ver comprometidos los niveles de excelencia deseados para los docentes formados en la Universidad de Chile).

- Propiciar una gestión académica moderna e integradora que posibilite el desarrollo de la unidad académica propuesta y su fluida articulación con las restantes unidades académicas de la Universidad, de manera indispensable e intensiva con aquellas que participan en la formación docente desde sus campos disciplinarios.

- Fortalecer la vinculación con el medio externo, potenciando los impactos de la Academia en el sistema educacional y su involucramiento en los problemas y desafíos del sistema escolar. La creación de esta unidad académica contribuirá al posicionamiento institucional de la Universidad de Chile en el campo de la educación, lo cual también facilitará el establecimiento de compromisos de largo plazo para colaborar con actores del sistema escolar. 
Asimismo, los cambios institucionales propuestos deberán irse acompañando de cambios en la cultura organizacional. En este marco, es posible pensar que la implementación del Proyecto Institucional de Educación de la Universidad de Chile aportará a esta un conjunto de externalidades positivas, tales como:

- Mayor flexibilización de los itinerarios curriculares en pregrado, a través de la promoción de una nueva relación entre el estudiante y la institución, más asociada al conjunto de la Universidad que a una unidad específica.

- Innovación en los mecanismos de selección de estudiantes.

- Mejor y mayor articulación de pregrado y postgrado.

- Mayor integración institucional en gestión y desarrollo académico, en especial por la vía de la vinculación entre unidades diferentes tras propósitos formativos comunes y proyectos compartidos.

- Modernización y profesionalización de la gestión universitaria, a través del desarrollo de capacidades para liderar y gestionar el cambio, promoción de la agilidad administrativa y foco en los objetivos académicos, entre otros.

- Vinculación con el medio para fines formativos, de investigación y de ejecución de proyectos de desarrollo colaborativo.

- Mayor internacionalización de la Universidad.

Así, las bases del modelo educativo para la formación de profesores desarrolladas en el marco de este proceso necesitan, por un lado, de condiciones como las aquí descritas y, por otro, aportarán desde su especificidad a la consolidación de cambios culturales relevantes para el futuro de la Universidad de Chile.

\section{Modelos de formación}

Las tres subcomisiones creadas para la investigación de la educación en cada uno de los niveles de parvularia, básica y media, después de analizar y debatir sobre los distintos modelos de formación, propusieron las orientaciones de base para los planes de formación inicial docente en cada uno de los tres niveles ya citados anteriormente.

\section{Educación Parvularia:}

La comisión FID (Formación Inicial Docente) de educación parvularia, coordinada por Marcela Pardo, analizó los criterios que deben regir para la admisión de los postulantes, las líneas gruesas para futuros planes de formación y perfiles de egreso, la formación pedagógica y la formación disciplinar de contenidos.

En cuanto a la formación disciplinar de contenidos se propone que la Universidad de Chile debe considerar las siguientes disciplinas para la formación de educadores de párvulos: Lenguaje, Artes, Matemáticas, Ciencias Naturales, Ciencias Sociales, Educación Física. 
La formación práctica constituye también un componente esencial en la educación de párvulos. Este espacio permite que las situaciones reales de docencia con niños se encuentren con la teoría.

Se propone que el componente de formación práctica incluya los tres niveles de la educación parvularia: 0-1 años, 2-3 años, 4-5 años, pues el trabajo de estos educadores varía significativamente con los rangos de edad de los alumnos.

\section{Educación Básica:}

A su vez, la comisión FID de Educación Básica, coordinada por Carmen Sotomayor, estableció los principios que deben orientar la formación de profesores en este nivel.

Las características principales de la propuesta son las siguientes:

Se propone crear una carrera de Educación Básica $\left(1^{\circ}\right.$ a $\left.6^{\circ}\right)$ de cinco años y de cobertura progresiva de manera que contribuya a mejorar la calidad de la formación de profesores de Educación Básica en el país.

Esta carrera debería caracterizarse por la alta especialización en las disciplinas básicas de Lenguaje, Matemática, Ciencias Sociales y Ciencias Naturales y en su enseñanza o didáctica específica. Además, se propone que la Educación Artística ocupe también un lugar y sea como un sello distintivo de la Universidad de Chile, a fin de no descuidar aspectos tan esenciales para el aprendizaje y desarrollo de los niños.

Junto a esta formación generalista de base, se propone que los estudiantes puedan formarse un año más en alguna especialización de estas áreas, profundizando en contenidos específicos.

Se pretende también superar la dicotomía entre formación disciplinaria y pedagógica. Todos los programas de países estudiados por la comisión -como Australia, Singapur o Estados Unidos- participan de esta tendencia. De modo que la propuesta del perfil del académico y de los futuros profesores y profesoras de Educación General Básica de la Universidad de Chile debe caracterizarse por una sólida formación disciplinaria y pedagógica, con capacidad de investigación y con un fuerte compromiso con la formación de profesores.

\section{Educación Media:}

La comisión FID de educación media, coordinada por Lino Cubillos, estableció, igualmente, los principales rasgos de la formación en el marco institucional con el fin de asegurar la necesaria coherencia de la propuesta con la misión y valores de la Universidad en relación a la formación de pregrado.

En este marco institucional, se examinó la documentación oficial, lo que permitió relevar importantes aspectos. El primero de ellos fija la orientación general de la Universidad respecto de la educación, donde aparece su responsabilidad impulsora del perfeccionamiento del sistema educacional del país. Un segundo aspecto tiene relación con los objetivos que la propia Universidad se plantea sobre la formación, específicamente sobre el compromiso de entregar a los jóvenes una formación integral para su desarrollo personal y profesional. 
Finalmente, el tercer elemento tiene relación con las orientaciones del Modelo Educativo de la Universidad. Este modelo es un documento orientador, fruto de los acuerdos y experiencia institucional que trata de reflejar el proceso de modernización de la formación de pregrado de los últimos años.

Las conclusiones de la comisión sobre el modelo de Formación Inicial de profesores de Educación Media en la Universidad de Chile, tiene en cuenta las experiencias habidas en la Universidad, especialmente en las facultades que actualmente imparten programas de Educación en Ciencias y en Humanidades y que, desde 1994, trabajan en la formación de profesores.

El modelo de formación pedagógica existente se basa en dos principios: enfoque investigativo y orientación al desempeño. En la actualidad, en la Universidad de Chile los profesores de Educación Media se forman en alguna de las siguientes modalidades.

a) Modelo post licenciatura: El profesor recibe formación en la disciplina que va a enseñar y de la que es especialista por razones de su licenciatura. Por tanto, lo que obtiene es una formación pedagógica profesional. Este modelo se conoce como consecutivo y es el modelo a través del cual la Universidad de Chile ha formado ya 17 promociones de profesores.

b) Como variante de esta modalidad, en 2005 se creó el programa de pedagogía en Física y Matemática, entre las facultades de Ciencias y de Filosofía y Humanidades. Se trata de un modelo de carácter bidisciplinario, basado en el consecutivo tradicional, pero con variantes que lo diferencian sustantivamente del modelo original: mantiene la formación disciplinaria durante los seis primeros semestres y en el séptimo y octavo semestre se realiza, parcialmente, la formación pedagógica en la propia facultad que imparte la disciplina de la Licenciatura en Ciencias. Es después de esta licenciatura cuando los estudiantes son transferidos la Facultad de Filosofía y Humanidades para recibir el programa de formación pedagógica, en su semestre noveno y décimo.

La conclusión de esta comisión fue, por tanto, que en Chile existen dos modelos de formación de docentes en enseñanza media: la formación concurrente y la formación consecutiva.

Habida cuenta de esta experiencia institucional y de la revisión de los modelos internacionales, la Comisión propone las siguientes modalidades de formación de profesores para la enseñanza media: Modelo Consecutivo, Modelo Doble Titulación y Modelo Consecutivo Integrado (o Semiconcurrente).

\section{El Modelo Consecutivo:}

Este modelo corresponde a la modalidad que ha desarrollado la Universidad durante los últimos años y que se acaba de exponer en los anteriores puntos. La comisión de Educación Media propone continuar y perfeccionar esta modalidad, por ser una modalidad de alta demanda por los alumnos y una fuente de buenos profesores para el sistema nacional. 


\section{El Modelo Doble Titulación:}

Esta modalidad mantiene cierta similitud con la licenciatura disciplinaria de formación pedagógica post-licenciatura, también expuesta en párrafos anteriores, pero debería ser ofertada con mayor flexibilidad. Estudiantes de cualquier carrera, con un componente disciplinario suficiente para una determinada área escolar (por ejemplo Medicina para poder impartir Biología) podrían tomar cursos del área pedagógica a lo largo de su carrera, como cursos electivos o complementarios. Estos cursos que toma por adelantado deberían servir como base para completar su formación pedagógica y obtener el título de profesor. Paralelamente, el estudiante obtendrá el título de la carrera que originalmente estaba siguiendo. La comisión propone explorar esta modalidad sobre la base de la experiencia que actualmente existe.

\section{Modelo Consecutivo Integrado (o Semiconcurrente):}

Esta modalidad tiene su expresión en la actual carrera de Pedagogía en Matemática y Física que implica el ingreso a la carrera docente desde el primer año. Se basa en una formación disciplinaria fuerte en los dos primeros años y se avanza en la formación pedagógica completa en los últimos años. Esta formación disciplinaria y pedagógica permite obtener la licenciatura en la disciplina correspondiente y la licenciatura en Educación, además del título de profesor en la disciplina o disciplinas que correspondan.

La comisión de Educación Media propone el desarrollo de esta modalidad en todas las disciplinas escolares (Matemática, Música, Lenguaje, etc.) de modo que esta se constituya en la componente central de la oferta formativa de profesores de enseñanza media.

A pesar de lo anterior, también existe un punto de experiencia: este modelo debería concentrar esfuerzos académicos de la Universidad que permitan desarrollar y perfeccionar la experiencia adquirida en la Pedagogía en Matemática y Física, extendiéndola a otras disciplinas.

\section{II. ¿DÓNDE ESTAMOS?}

Conociendo todos estos argumentos que acabamos de exponer, en el discurso de inauguración del año académico 2012, nos preguntábamos:

¿Dónde estamos? ¿Por qué la Universidad de Chile debiera aspirar hoy a involucrarse en educación a una escala académica y profesional mayor que la que hoy ya realiza? -mayor en calidad, cantidad, desarrollo, impacto, sustentabilidad y visibilidad-. ¿Para qué invertir tiempo y recursos de la Universidad y del país en un esfuerzo de esta naturaleza? ¿Acaso no nos basta con mantener o incrementar lo ya existente, mejorar los aspectos deficitarios más evidentes y conformarnos con lo que ya hemos logrado durante la última década? 
Además, afirmamos que la Universidad de Chile está y estará incompleta sin un área de educación fuerte y debidamente desarrollada al más alto nivel nacional e internacional, pero, igualmente reiteramos, este esfuerzo tendrá verdadero sentido y valor si somos capaces de realizarlo en colaboración con otros, especialmente a través de una interacción fecunda con otras universidades estatales que están trabajando por el mismo objetivo.

Al igual que lo que decíamos ayer, queremos seguir invitando hoy a la comunidad universitaria y a los órganos de gobierno universitario, a crear una Facultad de Educación en la Universidad de Chile. Sostenemos que el reto de toda nuestra comunidad universitaria, del Senado Universitario y del Consejo Universitario, será pensar una institucionalidad lo suficientemente imaginativa e innovadora de modo que la Facultad de Educación de la Universidad de Chile pueda hacer la diferencia; y que la potencie y le facilite la articulación de la educación con el resto de la Universidad, que haga que el proceso de desarrollar la educación en la Chile, así como la manera de formar nuevos profesores y de hacer investigación en Educación, sean un proceso eminentemente institucional, pues será la educación de la Universidad de Chile.

Estamos ciertos que para acercarnos a esa meta, durante los próximos dos años la Universidad de Chile debiera concretamente avanzar, tal como se ha propuesto en el documento Bases para el Proyecto Institucional de la Universidad de Chile, en:

- La creación de una nueva oferta académica de pregrado para la formación inicial docente que contemple nuevos programas en los niveles de educación parvularia, básica y media.

- Conformar una red de establecimientos educacionales de todos los tipos y niveles -empezando por nuestro Liceo Manuel de Salas, nuestro Instituto Artístico de Estudios Secundarios y, por supuesto, el Instituto Nacional, que este año cumple 200 años- con los que la Universidad establezca alianzas estratégicas con diferentes grados de profundidad y proyectos de desarrollo mutuo.

- En los próximos cuatro años, la matrícula total de programas de formación pedagógica debiera multiplicarse por tres llegando a cerca de 1.500 estudiantes inscritos en los diferentes programas, incluidos aquellos cuya tuición será compartida entre la Facultad de Educación y otras facultades.

- Siguiendo la experiencia acumulada hoy en el DEP, los sistemas de ingreso a los diferentes programas de formación docente considerarán otros criterios diferentes a la sola PSU, de manera tal de premiar aspectos vocacionales, habilidades específicas y el desempeño absoluto y relativo durante la enseñanza media.

- En investigación en educación y formación docente, potenciaremos el desarrollo y complementariedad de diferentes enfoques metodológicos -cualitativos y cuantitativos-, garantizando una presencia relevante de metodologías cualitativas y de investigaciones "situadas en la escuela", con intensa colaboración de los actores escolares, especialmente los docentes.

- En el posgrado diversificaremos, de manera colaborativa entre la Facultad de Educación y otras unidades, nuestra oferta de magísteres y postítulos. Al mismo tiempo, sentaremos las bases para ofrecer un doctorado de nivel internacional. 
Por su parte, la llamada formación docente continua deberá pasar a ser parte orgánica del proyecto académico de los programas de formación docente de pregrado, superando el reducido esquema de la venta de servicios y de la sola oferta de cursos de perfeccionamiento.

- Fortaleceremos el cuerpo académico a través de la contratación de un número significativo de nuevos académicos de jornada completa con doctorado y la conformación de equipos de trabajo capaces de liderar y abordar los nuevos desarrollos propuestos, con especial énfasis en el trabajo multidisciplinario. Para ello deberemos realizar una fase de transición que permita reestructurar las unidades actuales, alojando las nuevas contrataciones mientras se diseña y da forma a la implementación de la futura Facultad de Educación. Durante 2012 debiera constituirse un Instituto Transitorio de Educación y Formación Docente que permita asumir dichas tareas y abra el camino hacia las metas propuestas.

De este modo, podremos hacer real el anhelo de recuperar nuestro liderazgo en la educación y construir un horizonte de futuro para nuestra Universidad. Se trata de un proceso para dotar a la Universidad de Chile de un proyecto académico del cual hoy carece y cuyo potencial de innovación es enorme. Abordando seria y profundamente hoy los desafíos académicos de la educación, lo que la Universidad de Chile logrará es evolucionar ella misma hacia posibilidades insospechadas, con una mayor integración y coherencia, ofreciendo a Chile lo mejor de su antigua y nueva savia azul. 